\title{
Budget impact analysis resulting from the use of dabigatran etexilate in preventing stroke in patients with non-valvular atrial fibrillation in Italy
}

\author{
Francesco Saverio Mennini ${ }^{1,2}$, Sergio Russo ${ }^{1}$, Andrea Marcellusi ${ }^{1,3}$ \\ CEIS Sanità (CHEM - Centre for Health Economics and Management), Faculty of Economics, University of Rome "Tor Vergata", Italy \\ Department of Accounting and Finance at Kingston University, London, UK \\ Department of Statistics, University of Rome "La Sapienza", Italy
}

\begin{abstract}
Background: atrial fibrillation (AF) is the most common form of alteration in cardiac rhythm and associated with more severe episodes of stroke. Treatment with oral anticoagulants vitamin K antagonists (VKA) such as warfarin, is nowadays the therapy of choice for stroke prevention in patients with $\mathrm{AF}$, but dabigatran etexilate (DE) $150 \mathrm{mg}$ twice daily was more clinically effective than warfarin in reducing the risk of stroke or systemic embolism, ischaemic stroke and vascular mortality whereas DE $110 \mathrm{mg}$ twice daily was non-inferior to warfarin.

Aim: to assess the affordability of the use of DE for the Italian NHS, in patients with non-valvular AF (NVAF) through a budget impact analysis (BIA).

Methods: the BIA in a timeframe of 5 years was divided into 3 scenarios (1: current management of patients with NVAF; 2: all patients with NVAF treated with VKA; 3: all patients with NVAF treated with DE). The population considered is the one with indication for anticoagulation. Analysis is from the NHS perspective: therefore, indirect costs are excluded. Results: the underuse of oral anticoagulation, associated with the difficulty in keeping the patients treated with VKA within an acceptable therapeutic range, results in an enormous social and human cost, represented by a total of more than 63,000 strokes cumulated in the 5-year period considered. The cumulative cost for the scenario 1 over the 5 -year period is over $€$ 2.3 billion. In the scenario 2 the number of strokes avoided per year increases by $-5,219$ compared to no treatment $(-2,368$ compared to scenario 1), although the number of events remains high (about 10,000 events/year). In the 5-year observation period the scenario 2 would result in a reduction in the total number of strokes $(-12,323$ events vs. scenario 1$)$, and savings for the NHS of around 95 million Euros compared to scenario 1. In the Scenario 3 there is a reduction of more than 38,600 of the total cumulative number of strokes vs. the scenario 1 and over 26,200 vs. scenario 2, and savings for the NHS at the fifth year of observation of circa 174 million Euros vs. scenario 1 and 123 million Euros vs. scenario 2.

Conclusion: DE in Italy is economically sustainable, as it allows savings for the NHS in the management of patients with NVAF from the second year vs. no treatment and vs. treatment with VKA.
\end{abstract}

Keywords

Dabigatran etexilate; Vitamin K antagonists; Non-valvular atrial fibrillation; Budget impact analysis

\section{INTRODUCTION}

Atrial fibrillation $(\mathrm{AF})$ is the most common form of alteration in cardiac rhythm, with a prevalence of $1-2 \%$ in the general population [1]. AF, even in its non-valvular form (NVAF), is associated with more severe episodes of stroke, with a higher degree of residual disability, or associated with increased mortality, particularly in patients aged $\geq 75$ years [2]. In Western countries, the annual cost of treatment for patient with $\mathrm{AF}$ is estimated to be about $€ 3,000$ [3], which projected on the Italian population $(543,000$ subjects with NVAF in 2011) causes a potential expenditure of $€ 1,629,000,000$ (about $2 \%$ of total public health expenditure). This expenditure is mainly due to the number of events caused by AF (stroke, intracranial and extracranial hemorrhages, transient ischemic attacks, myocardial infarction, etc.). Of these, the stroke is the most important event in terms of economic impact for the National Health Service (NHS), being the third leading cause of death (after cardiovascular diseases and cancer) and the leading cause of long-term serious disability [4].
Corresponding author Prof. Francesco Saverio Mennini f.mennini@uniroma2.it

\section{Disclosure}

Editorial support was provided by an unrestricted grant from Boehringer Ingelheim Italy 
In Italy there are about 200,000 people affected by stroke every year: of these, 40,000 die shortly after and another 40,000 lose completely their self-sufficiency, completely changing their lives and those of their families [4]. It is estimated that $15 \%$ of total stroke is attributable to subjects with AF (about 30,000 strokes) [4]. Given these events, the cost to the NHS per stroke is about $€ 17,500$, the annual total cost $€ 3,500,000,000$ for all strokes and $€ 525,000,000$ for those caused by AF [4]. Treatment with oral anticoagulants vitamin $\mathrm{K}$ antagonists (VKA) such as warfarin, is nowadays the therapy of choice for stroke prevention in patients with atrial fibrillation [5]. However, the difficulty in maintaining the anticoagulant effect in the therapeutic range greatly limits its effectiveness, increasing the probability of bleeding, as well as representing a contraindication to the prescription of therapy in patients with poor compliance or unable to perform the necessary laboratory checks. In addition, many patients who are administered this therapy, if not followed in specialized centers (TAO - anticoagulation therapy center) may suffer from inadequate anticoagulation [6]. In the RE-LY study (Randomized Evaluation of Long-Term Anticoagulation Therapy), dabigatran etexilate has proved effective as warfarin at the lowest dose (110 mg twice daily) and significantly more effective than warfarin at the highest dose (150 mg twice daily) in preventing stroke and systemic embolisms over a two-year period of treatment in patients with atrial fibrillation and risk of stroke [7].

\section{AIM OF THE STUDY}

The study aims to assess the affordability of the use of dabigatran etexilate (DE) for the Italian NHS, in patients with NVAF through a budget impact analysis in a timeframe of 5 years.

\section{METHODS}

Scenarios and reference population

The budget impact analysis in a timeframe of 5 years was divided into 3 scenarios:

- Scenario 1: current management of patients with NVAF.

- Scenario 2: all patients with NVAF treated with VKA.

- Scenario 3: all patients with NVAF treated with DE.

The population considered is the one with indication for anticoagulation. Therefore, also patients intolerant of VKA and patients with severe renal impairment are included.
Both types of patients are distributed evenly (VKA-treated patients and patients treated with dabigatran etexilate) among the various scenarios, avoiding biases regarding the comparison among the budget impacts.

\section{Assumptions of the analysis}

This was an analysis from the Italian NHS perspective: therefore, indirect costs are excluded, although they represent a major economic burden, in the case of warfarin use, both for families and society. Moreover, for estimating the target population of the analysis and the associated costs, the following assumptions are considered:

- In Italy, NVAF patients with indication for anticoagulation therapy are 335,000 (Appendix A).

- The risk of stroke is $4.5 \%$ per year in patients with untreated NVAF [8].

- The reduction in the risk of ischemic stroke associated with the treatment with warfarin, at adjusted dosage in order to achieve INR values between 2 and 3 , is $65 \%$ [9].

- The incidence of hemorrhagic stroke with warfarin, well controlled, is equal to 0.38 x 100 patients/year [7,10].

- The average time in therapeutic range (TTR) in Italy for patients treated with VKA is equal to $56.3 \%$ [11].

- The odds of ischemic events is $43 \%$ in patients treated with warfarin with TTR $<60 \%$ [12].

- The average cost of management of acute stroke is equal to $17,500 € /$ year [4].

- The average cost of managing post-stroke period is equal to $7,600 € /$ year [13].

- The costs of management of stroke here considered may be conservative, since it is known that the atrial fibrillation-related strokes are generally more severe and disabling [4].

- The average cost of INR is equal to 355 $€ /$ year (LR Veneto data [14] updated to 2010, Istat index).

- The cost of warfarin is $0.05 € /$ day (16.2 $€ /$ year).

- The assumed cost of dabigatran etexilate is $2.10 € /$ day (current price in Spain; today, May 2012, the lowest price in Europe).

- Patients treated with dabigatran etexilate undergo creatinine clearance tests (kidney check-up). We assume once a year (cost $=1.80 € /$ service according to Regional Tariff).

- There is an increase in the number of patients with NVAF in the considered years of $2 \%$ per year [15], even after considering a $12 \%$ mortality of the 
population (about twice the mortality of the general population in age-matched groups, as indicated in the 2010 ESC guidelines [16]).

- Dabigatran etexilate $150 \mathrm{mg}$ causes a $75 \%$ reduction in stroke risk compared with no treatment $(\mathrm{RR}=0.25 ; 95 \% \mathrm{CI}$ : 0.12-0.51) [17].

- Dabigatran etexilate $110 \mathrm{mg}$ results in a $65 \%$ reduction in stroke risk compared with no treatment $(\mathrm{RR}=0.35 ; 95 \% \mathrm{CI}$ : 0.17-0.71) [17].

- The incidence of hemorrhagic stroke with dabigatran etexilate is $0.11 \times 100$ patients/year in the RE-LY study [7,10]. In Europe, regulatory authorities recommend the use of dabigatran etexilate 150 $\mathrm{mg}$ twice daily for patients younger than 80 years (approximately $55,8 \%$ of the total population eligible to treatment) and $110 \mathrm{mg}$ twice daily for patients aged $\geq 80$ years (approximately 55,8\% of the total population eligible to treatment).

In order to simplify the comparison, it was assumed that:

- In every scenario, all patients are treated from the first day of every year, to assess the maximum potential impact of the various costs.

- The adherence to the treatment for dabigatran etexilate and warfarin is $95 \%(728 € /$ year and $16,2 € /$ year, respectively) $[7,10]$.

- The datum on the time in therapeutic range $(2 \leq \mathrm{INR} \geq 3)$ used is that for the patients treated for more than 1 year. This is justified by the fact that international and national studies show that, for patients treated for less than 1 year, the TTR is significantly lower than the TTR correlated with a longer duration of anticoagulant treatment, remaining below the average figure of $50 \%[4,11]$. In fact, as widely recognized by the international scientific community [3], these patients are at significantly greater risk of developing a stroke than others. On the other hand, the inclusion of this parameter in scenarios 1 and 2 would have lead to a considerable increase in the hypothetical cost of disease management or an advantage in the scenario where dabigatran etexilate is used. It was therefore decided to prefer the simplicity of analysis of the model and not to include this additional parameter.

\section{Sensitivity analysis}

Finally, a one-way sensitivity analysis was performed on the costs of monitoring patients on VKA therapy and on the costs of the drug; both parameters, taken into ac- count, are those most affected by a high variability (heterogeneity of monitoring costs), uncertainty (the price of the drug will be determined by AIFA) and impact on the final budget impact result. The minimum $(€ 212)$ and maximum ( $€$ 446) value of the costs of treatment monitoring of patients on VKA therapy was extracted from a research done by ANMDO (2012) in collaboration with the Centro Interdipartimentale di Studi Internazionali sull'Economia e lo Sviluppo (CEIS) at the Faculty of Economics, University of Rome Tor Vergata, and the Centro di Ricerche e Studi in Management Sanitario (CERISMAS) at the Catholic University of Sacro Cuore in Rome. Variations of $+/-10 \%$ were applied to the cost of the drug.

\section{RESULTS}

\section{Scenario 1: current management of patients with NVAF}

In the year 1 (2011), 335,000 individuals with NVAF have indication for oral anticoagulation, of whom approximately 183,000 are treated with VKA, while the remaining 152,000 aren't treated with oral anticoagulants. In Table I the current situation versus no treatment is analyzed in terms of events prevented by the current management of disease (year 1). Table II shows the 5-year prediction of ischemic and hemorrhagic strokes in the Italian situation.

From Table I it is clear how the current treatment of NVAF involves the reduction of only 2,851 strokes, in the first year of observation, compared with the total number of strokes developed in the scenario of total non-treatment $(15,075)$. This confirms that, despite the enormous organizational effort, there is still a major unmet need in the management of NVAF. The underuse of oral anticoagulation, associated with the difficulty in keeping the patients treated with VKA within an acceptable therapeutic range, results in an enormous social and human cost, represented by a total of more than 63,000 strokes cumulated in the 5-year period considered (Table II).

Starting from the cost assumptions reported in the background when presenting the scenarios, Table III shows the current cost of management of NVAF. Table III shows that the cumulative cost over the 5-year period is over $€ 2.3$ billions and the major cost generator in the management of NVAF is the cost of stroke management ( $€ 2.03$ billions). Indirect costs, burdening families and society, were not considered in this analysis. According to the CENSIS survey, presented in 2011 


\begin{tabular}{|c|c|c|}
\hline \multicolumn{3}{|l|}{ No treatment } \\
\hline Total patients with NVAF (nr.) & \multicolumn{2}{|c|}{335,000} \\
\hline Risk of stroke (\%/year) & \multicolumn{2}{|c|}{4.50} \\
\hline Total strokes (nr./year) & \multicolumn{2}{|c|}{15,075} \\
\hline \multicolumn{3}{|c|}{ Current situation } \\
\hline & Pts treated with VKA & Pts not treated with VKA \\
\hline Total patients with NVAF (nr.) & 183,000 & 152,000 \\
\hline Number of strokes (nr./year) & 8,235 & 6,840 \\
\hline VKA: risk reduction with "real life"-TTR of 56.3\% (\%) & -43 & 0 \\
\hline Ischemic strokes (nr./year) & 4,694 & 6,840 \\
\hline Total ischemic strokes (nr./year) & \multicolumn{2}{|c|}{11,534} \\
\hline Hemorrhagic strokes (nr./year) & 690 & $0^{*}$ \\
\hline Total hemorrhagic strokes (nr./year) & \multicolumn{2}{|c|}{690} \\
\hline Total ischemic/hemorrhagic strokes (nr./year) & \multicolumn{2}{|c|}{12,224} \\
\hline Total ischemic/hemorrhagic strokes prevented vs. no treatment (nr./year) & \multicolumn{2}{|c|}{2,851} \\
\hline
\end{tabular}

Table I. Current situation vs. no treatment (year 1)

* The figure of hemorrhagic strokes (total per year), in the group of patients not treated with VKA, has been considered to be zero to simplify the calculation

\begin{tabular}{lcccccc}
\hline & Year $\mathbf{1}$ & Year $\mathbf{2}$ & Year 3 & Year 4 & Year 5 & Total \\
\hline Events in no treatment (nr.) & 15,075 & 15,377 & 15,684 & 15,998 & 16,318 & 78,452 \\
Events in current situation (nr.) & 12,224 & 12,468 & 12,718 & 12,972 & 13,232 & 63,614 \\
Prevented events current situation vs. no treatment (nr.) & 2,851 & 2,909 & 2,966 & 3,026 & 3,086 & 14,838 \\
\hline
\end{tabular}

Table II. Prevented events (ischemic and hemorrhagic strokes), current situation vs. no treatment over 5 year

\begin{tabular}{lcccccc}
\hline \multicolumn{1}{c}{ Cost item } & Year 1 & Year $\mathbf{2}$ & Year $\mathbf{3}$ & Year 4 & Year 5 & Total \\
\hline INR monitoring $(€ \times 1,000)$ & 64,965 & 6,264 & 67,590 & 68,941 & 70,320 & 338,080 \\
VKA $(€ \times 1,000)$ & 2,965 & 3,024 & 3,084 & 3,146 & 3,209 & 15,428 \\
Management of ischemic/hemorrhagic stroke $(€ \times 1,000)$ & 213,920 & 311,228 & 406,259 & 503,086 & 601,745 & $2,036,237$ \\
Total cost $(€ \times 1,000)$ & 281,850 & 380,516 & 476,933 & 575,173 & 675,274 & $2,389,745$ \\
\hline
\end{tabular}

Table III. Current cost of management of NVAF in the scenario 1 (current situation)

at the World Day of stroke, every stroke patient costs about $€ 30,000$ to the family per year [18].

\begin{tabular}{|c|c|}
\hline \multicolumn{2}{|l|}{ No treatment } \\
\hline Total patients with NVAF (nr.) & 335,000 \\
\hline Risk of stroke (\%/year) & 4.50 \\
\hline Total strokes (nr./year) & 15,075 \\
\hline \multicolumn{2}{|l|}{ Pts treated with VKA } \\
\hline Total patients with NVAF (nr.) & 335,000 \\
\hline VKA: risk reduction with "real life"-TTR of $56.3 \%$ & $-43 \%$ \\
\hline Total observed ischemic strokes (nr./year) & 8,593 \\
\hline Total hemorrhagic strokes (nr./year)* & 1,263 \\
\hline Total ischemic/hemorrhagic strokes (nr./year) & 9,856 \\
\hline $\begin{array}{l}\text { Total ischemic/hemorrhagic strokes prevented vs. no treatment } \\
\text { (nr./year) }\end{array}$ & 5,219 \\
\hline
\end{tabular}

Table IV. Patients treated with VKA vs. no treatment (year 1)

* $0.38 \%$ per year

\section{Scenario 2: all patients with NVAF treated with VKA}

Scenario 2 was developed on the assumption that all patients with NVAF are treated with VKA. This is a hypothetical scenario, since there are both structural and therapeutic limitations. Structural limitations due to the uneven presence of anticoagulation therapy centers on the Italian territory, despite such therapy is available for over 50 years. Therapeutic limitations due to:

- Resistance to the use of VKA for fear of bleeding.

- Difficult management of this therapy, making more than $30 \%$ of patients treated with VKA discontinue therapy after one year.

- Difficulty in maintaining the patients treated with VKA within an acceptable TTR. 


\begin{tabular}{lcccccc}
\hline & Year $\mathbf{1}$ & Year $\mathbf{2}$ & Year $\mathbf{3}$ & Year $\mathbf{4}$ & Year $\mathbf{5}$ & Total \\
\hline Events in no treatment (nr.) & 15,075 & 15,377 & 15,684 & 15,998 & 16,318 & 78,452 \\
Events in VKA treatment (nr.) & 9,856 & 10,053 & 10,254 & 10,459 & 10,668 & 51,290 \\
Prevented events VKA vs. no treatment (nr.) & 5,219 & 5,323 & 5,430 & 5,539 & 5,649 & 27,160 \\
\hline
\end{tabular}

Table V. Prevented events (ischemic and hemorrhagic strokes) VKA treatment vs. no treatment over 5 year

\begin{tabular}{lcccccc}
\hline \multicolumn{1}{c}{ Cost item } & Year 1 & Year 2 & Year 3 & Year 4 & Year 5 & Total \\
\hline INR monitoring $(€ \times 1,000)$ & 118,925 & 121,304 & 123,730 & 126,204 & 128,728 & 618,890 \\
VKA $(€ \times 1,000)$ & 5,427 & 5,536 & 5,646 & 5,759 & 5,874 & 28,242 \\
Management of ischemic/hemorrhagic stroke $(€ \times 1,000)$ & 172,479 & 250,928 & 329,447 & 408,035 & 486,696 & $1,647,585$ \\
Total cost $(€ \times 1,000)$ & 296,831 & 377,767 & 458,823 & 539,999 & 621,298 & $2,294,718$ \\
\hline
\end{tabular}

Table VI. Cost of patients treated with VKA vs. no treatment over 5 years

\begin{tabular}{lcccccc}
\hline \multicolumn{1}{c}{ Scenario 2 vs. scenario 1 } & Year 1 & Year $\mathbf{2}$ & Year $\mathbf{3}$ & Year $\mathbf{4}$ & Year $\mathbf{5}$ & Total \\
\hline Ischemic strokes $(\mathrm{nr}$.) & $-2,941$ & $-3,000$ & $-3,060$ & $-3,121$ & $-3,184$ & $-15,306$ \\
Hemorrhagic strokes $(\mathrm{nr}$.) & 573 & 585 & 596 & 608 & 620 & 2,983 \\
Total ischemic/hemorrhagic strokes $(\mathrm{nr})$. & $-2,368$ & $-2,415$ & $-2,464$ & $-2,513$ & $-2,563$ & $-12,323$ \\
Cost of INR monitoring $(€ \times 1,000)$ & 53,960 & 55,039 & 56,140 & 57,263 & 58,408 & 280,810 \\
Drug cost $(€ \times 1,000)$ & 2,462 & 2,512 & 2,562 & 2,613 & 2,665 & 12,814 \\
Cost of management of ischemic/hemorrhagic stroke & $-41,441$ & $-60,299$ & $-76,812$ & $-95,051$ & $-115,049$ & $-388,651$ \\
$(€ \times 1,000)$ & & & & & & \\
Total cost $(€ \times 1,000)$ & 14,982 & $-2,748$ & $-18,110$ & $-35,175$ & $-53,975$ & $-95,027$ \\
\hline
\end{tabular}

Table VII. VKA treatment of all patients vs. current situation over 5 years

Table IV lists the number of ischemic and hemorrhagic strokes resulting from this scenario, and how many of them would be avoided in comparison with the current situation. Table $\mathrm{V}$ shows the prediction of the 5-year period of ischemic and hemorrhagic strokes after treatment with VKA of the entire Italian population with NVAF.

In this scenario (Table IV), the number of strokes avoided per year increases to $-5,219$ compared to no treatment $(-2,368$ compared to the current situation), although the number of events remains high (about 10,000 events/ year; Table V). Starting from the cost assumptions reported in methods section, Table VI shows the cost of management of NVAF if all patients were treated with VKA. Again, as in the previous scenario, the cumulative cost over the 5-year period is over 2 billion Euros (2.294 billion Euros).

Although in the presence of a theoretical extensive use of the VKA on all patients, the difficulty of maintaining within an acceptable therapeutic range the patients treated with VKA imposes an enormous social and human cost, represented by a total of approximately
51,000 strokes cumulated in the 5-year period under analysis.

The Table VII shows that an extensive use of VKA, scenario 2, in the 5-year observation period would result in:

- A reduction in the total number of strokes $(12,323$ fewer events in scenario 2 than in scenario 1).

- Savings for the NHS of around 95 million Euros compared to scenario 1.

\section{Scenario 3: all patients with NVAF treated with dabigatran etexilate}

Scenario 3: all patients with NVAF treated with dabigatran etexilate Tables VIII-IX show the potential ischemic and hemorrhagic strokes when using dabigatran etexilate on the entire population with NVAF.

Tables VIII and IX show that:

- The total number of strokes per year is significantly reduced compared with no treatment (about 4,800 strokes compared with about 15,075 of no treatment);

- The number of strokes avoided per year is about 7,500 compared to scenario 1 and about 5,000 compared to scenario 2 . 


\begin{tabular}{|c|c|c|}
\hline \multicolumn{3}{|c|}{ No treatment } \\
\hline Total patients with NVAF (nr.) & \multicolumn{2}{|c|}{335,000} \\
\hline Risk of stroke (\%/year) & \multicolumn{2}{|c|}{4.50} \\
\hline Total strokes (nr./year) & \multicolumn{2}{|c|}{15,075} \\
\hline \multicolumn{3}{|c|}{ Pts treated with DE } \\
\hline & DE $150 \mathrm{mg}$ (pts $<80$ years) & DE $110 \mathrm{mg}$ (pts $\geq 80$ years) \\
\hline Patients with NVAF (nr.) & 187,000 & 148,000 \\
\hline DE: reduction in the risk of stroke (\%) & -75 & -65 \\
\hline Ischemic strokes (nr./year) & 2,059 & 2,394 \\
\hline Total ischemic strokes (nr./year) & \multicolumn{2}{|c|}{4,435} \\
\hline Hemorrhagic strokes (nr./year) & 205 & 163 \\
\hline Total hemorrhagic strokes (nr./year) & \multicolumn{2}{|c|}{368} \\
\hline Total ischemic/hemorrhagic strokes (nr./year) & \multicolumn{2}{|c|}{4,803} \\
\hline $\begin{array}{l}\text { Total ischemic/hemorrhagic strokes prevented vs. no treatment } \\
\text { (nr./year) }\end{array}$ & \multicolumn{2}{|c|}{10,272} \\
\hline
\end{tabular}

Table VIII. Patients treated with dabigatran etexilate (DE) vs. no treatment (year 1)

\begin{tabular}{lcccccc}
\hline & Year 1 & Year $\mathbf{2}$ & Year 3 & Year 4 & Year 5 & Total \\
\hline Events in no treatment (nr.) & 15,075 & 15,377 & 15,684 & 15,998 & 16,318 & 78,452 \\
Events in DE treatment (nr.) & 4,803 & 4,899 & 4,997 & 5,097 & 5,199 & 24,995 \\
Prevented events DE vs. no treatment (nr.) & 10,272 & 10,478 & 10,687 & 10,901 & 11,119 & 53,457 \\
\hline
\end{tabular}

Table IX. Prevented events (ischemic and hemorrhagic strokes): treatment with dabigatran etexilate (DE) over 5 years

\begin{tabular}{lcccccc}
\hline \multicolumn{1}{c}{ Cost item } & Year 1 & Year 2 & Year 3 & Year 4 & Year 5 & Total \\
\hline $\mathrm{DE}^{*}(€ \times 1,000)$ & 244,542 & 249,432 & 254,421 & 259,510 & 264,700 & $1,272,604$ \\
Management cost of ischemic + hemorrhagic stroke $(€ \times 1,000)$ & 84,047 & 122,153 & 160,289 & 198,458 & 236,658 & 801,605 \\
Total cost $(€ \times 1,000)$ & 328,589 & 371,585 & 414,710 & 457,967 & 501,358 & $2,074,209$ \\
\hline
\end{tabular}

Table X. Cost of patients treated with dabigatran etexilate (DE)

${ }^{*}$ Cost $/$ day $=€ 2.10$; Compliance rate $95 \%$

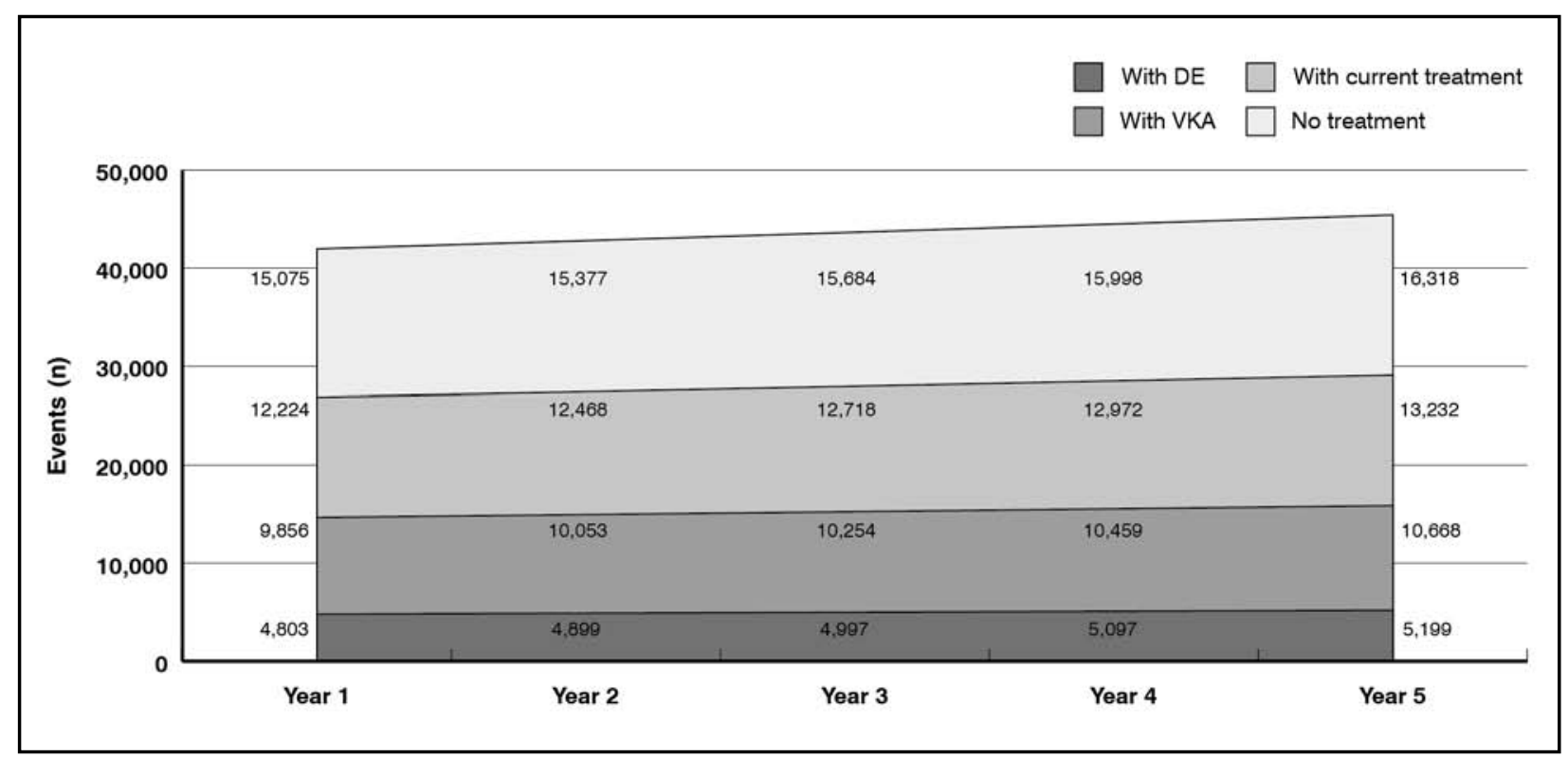

Figure 1.Number of events (hemorrhagic and ischemic strokes) in the various scenarios $\mathrm{DE}=$ dabigatran etexilate 
Similarly to the previous scenarios, starting from the cost assumptions reported in the background, and using a daily cost of dabigatran etexilate equal to $€ 2.10$ (the lowest in Europe in May 2012: current price in Spain), Table $\mathrm{X}$ reports the management costs of NVAF. Figures 1, 2 and 3 show the comparison among the scenario 3 (all the patients treated with dabigatran etexilate) and other scenarios in terms of events and costs.

An extensive use of dabigatran etexilate would cause:

- A reduction of more than 38,500 of the total cumulative number of strokes vs. the current scenario and over 26,297 vs. the treatment with VKA, with all the obvious social and health benefits in terms of health "saved".

- An increase in the cost of drug treatment, which in this case must be considered as the potential maximal expenditure.

- Savings for the NHS at the fifth year of observation of 173 million Euros vs. the current treatment and 123 million Euros vs. treatment with VKA.

The savings from the reduction of indirect costs of both society and families of patients with NVAF should be added to all this, resulting in huge direct and indirect savings for the society.

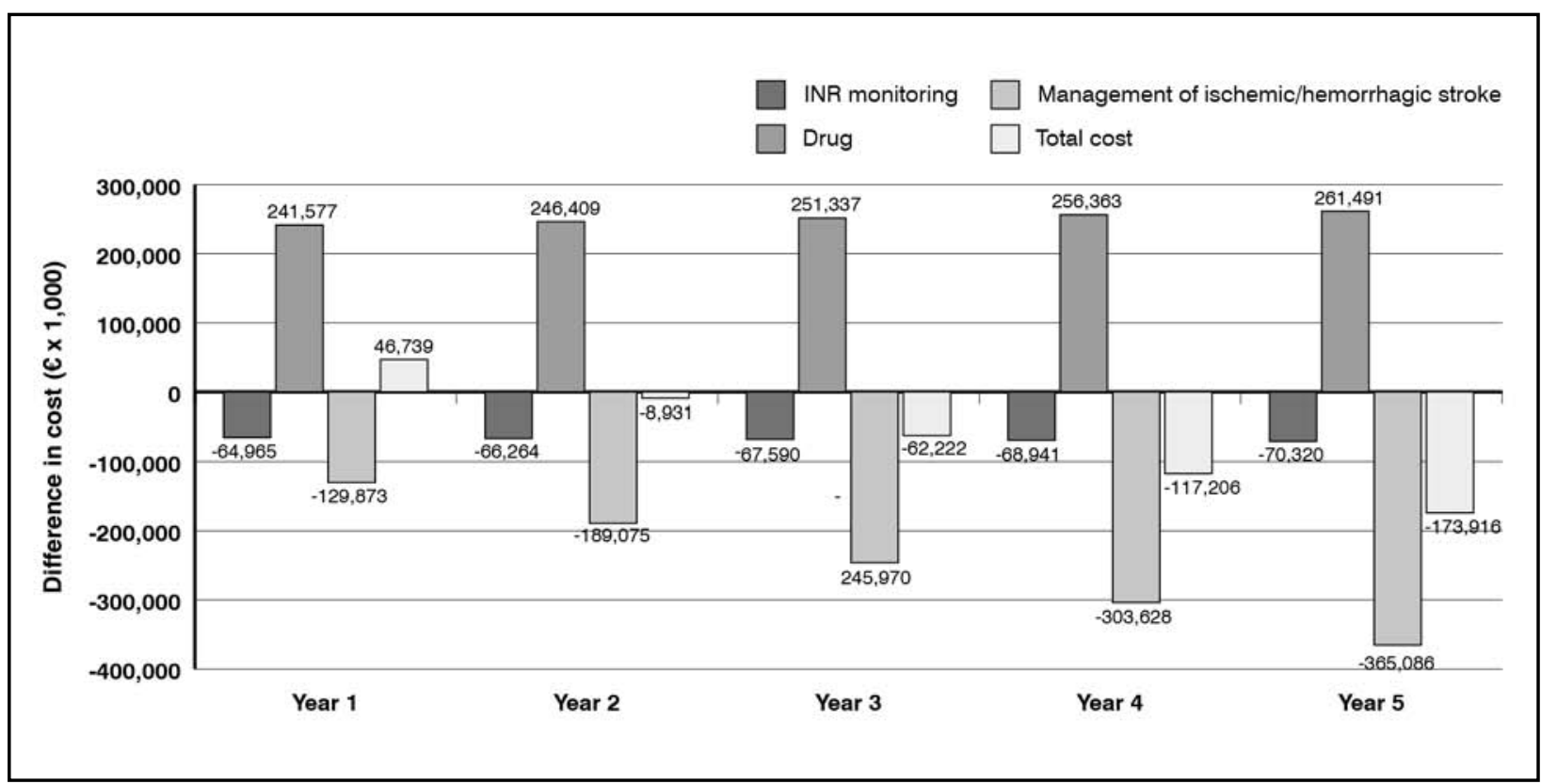

Figure 2.Differences in cost of dabigatran etexilate vs. current scenario

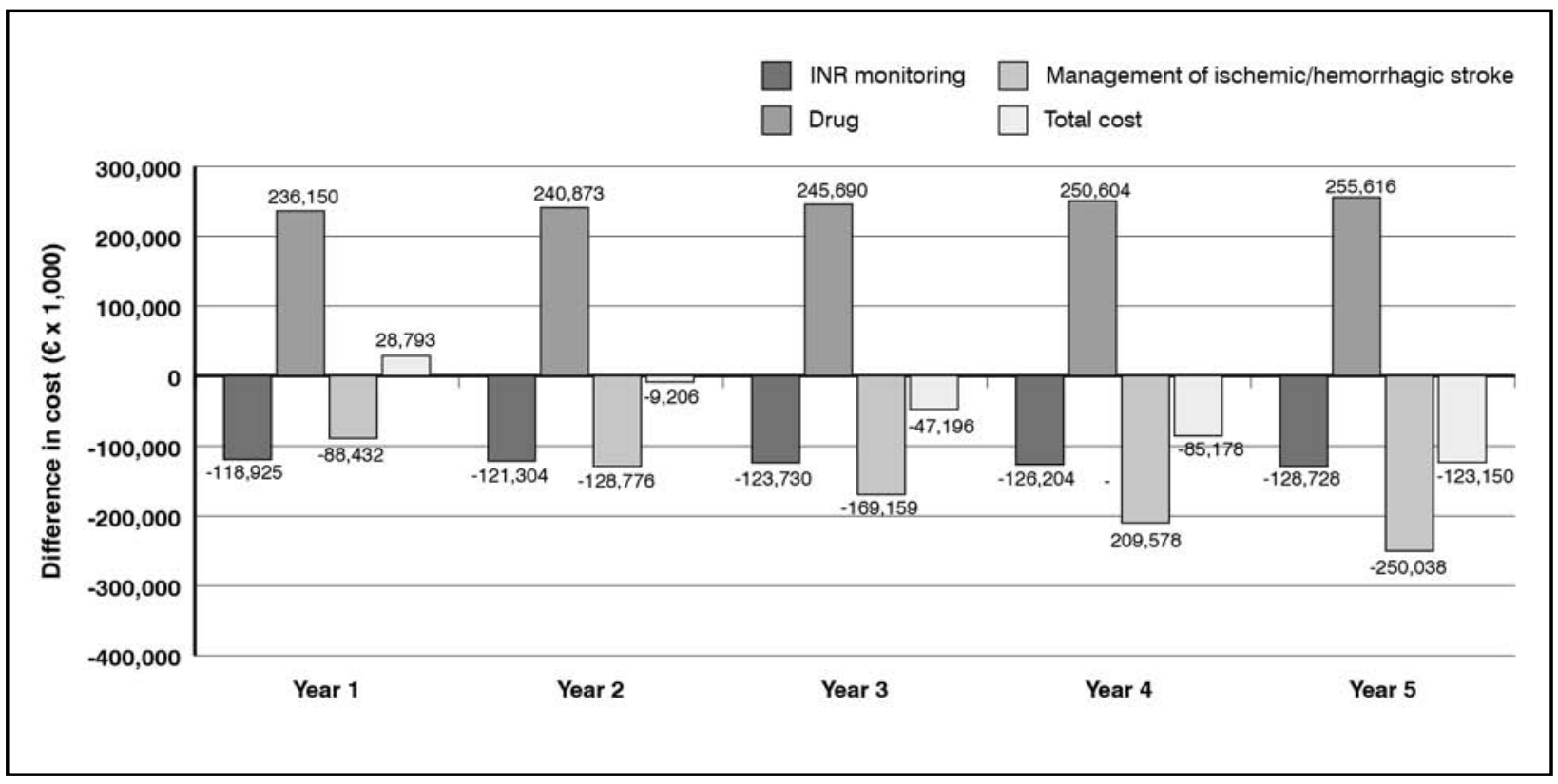

Figure 3. Differences in cost of dabigatran etexilate vs. treatment with VKA 


\begin{tabular}{lcccccc}
\hline \multicolumn{1}{c}{ Scenario $\mathbf{3}$ vs. scenario 2 } & Year $\mathbf{1}$ & Year $\mathbf{2}$ & Year $\mathbf{3}$ & Year $\mathbf{4}$ & Year $\mathbf{5}$ & Total \\
\hline Ischemic strokes $(\mathrm{nr}$.) & $-4,158$ & $-4,241$ & $-4,326$ & $-4,413$ & $-4,501$ & $-21,638$ \\
Hemorrhagic strokes $(\mathrm{nr}$.) & -895 & -913 & -931 & -950 & -969 & $-4,659$ \\
Total ischemic/hemorrhagic strokes $(\mathrm{nr})$. & $-5,053$ & $-5,154$ & $-5,257$ & $-5,363$ & $-5,470$ & $-26,297$ \\
Cost of INR monitoring $(€ \times 1,000)$ & $-71,020$ & $-72,440$ & $-73,889$ & $-75,367$ & $-76,874$ & $-369,591$ \\
Drug cost $(€ \times 1,000)$ & 236,150 & 240,873 & 245,690 & 250,604 & 255,616 & $1,228,934$ \\
Cost of management of ischemic/hemorrhagic stroke & $-88,432$ & $-128,776$ & $-169,157$ & $-209,578$ & $-250,038$ & $-845,980$ \\
$(€ \times 1,000)$ & & & & & & \\
Total cost $(€ \times 1,000)$ & 76,698 & 39,657 & 2,644 & $-34,341$ & $-71,296$ & 13,363 \\
\hline
\end{tabular}

Table XI. Dabigatran etexilate vs. treatment with VKA (cost of monitoring $=€ 212$ )

\begin{tabular}{|c|c|c|c|c|c|c|}
\hline Scenario 3 vs. scenario 2 & Year 1 & Year 2 & Year 3 & Year 4 & Year 5 & Total \\
\hline Ischemic strokes (nr.) & $-4,158$ & $-4,241$ & $-4,326$ & $-4,413$ & $-4,501$ & $-21,638$ \\
\hline Hemorrhagic strokes (nr.) & -895 & -913 & -931 & -950 & -969 & $-4,659$ \\
\hline Total ischemic/hemorrhagic strokes (nr.) & $-5,053$ & $-5,154$ & $-5,257$ & $-5,363$ & $-5,470$ & $-26,297$ \\
\hline Cost of INR monitoring $(€ \times 1,000)$ & $-149,410$ & $-152,398$ & $-155,446$ & $-158,555$ & $-161,726$ & $-777,536$ \\
\hline Drug cost $(€ \times 1,000)$ & 236,150 & 240,873 & 245,690 & 250,604 & 255,616 & $1,228,934$ \\
\hline $\begin{array}{l}\text { Cost of management of ischemic/hemorrhagic stroke } \\
(€ \times 1,000)\end{array}$ & $-88,432$ & $-128,776$ & $-169,157$ & $-209,578$ & $-250,038$ & $-845,980$ \\
\hline Total cost $(€ \times 1,000)$ & $-1,692$ & $-40,301$ & $-78,913$ & $-117,529$ & $-156,148$ & $-394,582$ \\
\hline
\end{tabular}

Table XII. Dabigatran etexilate vs. VKA treatment (cost of monitoring $=€ 446$ )

\begin{tabular}{|c|c|c|c|c|c|c|}
\hline Scenario 3 vs. scenario 2 & Year 1 & Year 2 & Year 3 & Year 4 & Year 5 & Total \\
\hline Ischemic strokes (nr.) & $-4,158$ & $-4,241$ & $-4,326$ & $-4,413$ & $-4,501$ & $-21,638$ \\
\hline Hemorrhagic strokes (nr.) & -895 & -913 & -931 & -950 & -969 & $-4,659$ \\
\hline Total ischemic/hemorrhagic strokes (nr.) & $-5,053$ & $-5,154$ & $-5,257$ & $-5,363$ & $-5,470$ & $-26,297$ \\
\hline Cost of INR monitoring $(€ \times 1,000)$ & $-118,925$ & $-121,304$ & $-123,730$ & $-126,204$ & $-128,728$ & $-618,890$ \\
\hline Drug cost $(€ \times 1,000)$ & 211,756 & 215,991 & 220,311 & 224,717 & 229,212 & $1,101,988$ \\
\hline $\begin{array}{l}\text { Cost of management of ischemic/hemorrhagic stroke } \\
(€ \times 1,000)\end{array}$ & $-88,432$ & $-128,776$ & $-169,157$ & $-209,578$ & $-250,038$ & $-845,980$ \\
\hline Total cost $(€ \times 1,000)$ & 4,399 & $-34,088$ & $-72,576$ & $-111,065$ & $-149,554$ & $-362,883$ \\
\hline
\end{tabular}

Table XIII. Dabigatran etexilate $(1,89 € /$ die) vs. VKA treatment

\begin{tabular}{|c|c|c|c|c|c|c|}
\hline Scenario 3 vs. scenario 2 & Year 1 & Year 2 & Year 3 & Year 4 & Year 5 & Total \\
\hline Ischemic strokes (nr.) & $-4,158$ & $-4,241$ & $-4,326$ & -4.413 & $-4,501$ & $-21,638$ \\
\hline Hemorrhagic strokes (nr.) & -895 & -913 & -931 & -950 & -969 & $-4,659$ \\
\hline Total ischemic/hemorrhagic strokes (nr.) & $-5,053$ & $-5,154$ & $-5,257$ & $-5,363$ & $-5,470$ & $-26,297$ \\
\hline Cost of INR monitoring $(€ \times 1,000)$ & $-118,925$ & $-121,304$ & $-123,730$ & $-126,204$ & $-128,728$ & $-618,890$ \\
\hline Drug cost $(€ \times 1,000)$ & 260,544 & 265,755 & 271,070 & 276,491 & 282,021 & $1,355,881$ \\
\hline $\begin{array}{l}\text { Cost of management of ischemic/hemorrhagic stroke } \\
(€ \times 1,000)\end{array}$ & $-88,432$ & $-128,776$ & $-169,157$ & $-209,578$ & $-250,038$ & $-845,980$ \\
\hline Total cost $(€ \times 1,000)$ & 53,187 & 15,676 & $-21,817$ & $-59,291$ & $-96,745$ & $-108,990$ \\
\hline
\end{tabular}

Table XIV. Dabigatran etexilate vs. VKA treatment 


\section{SENSITIVITY ANALYSIS}

\section{Cost of monitoring of the patient receiving VKA therapy $=€ 212$}

As showed in Table XI an extensive use of dabigatran etexilate would result in:

- Also in this situation, a saving for the NHS at the fifth year of observation of 71 million Euros vs. the treatment with VKA (-43\% compared to the basal hypothesis).

- A reduction of 26,297 in the total number of cumulative strokes vs. the treatment with VKA, with all the obvious social and health benefits in terms of health "saved" (savings in terms of clinical benefit remain unchanged).

\section{Cost of monitoring of the patient receiving VKA therapy $€ \mathbf{4 4 6}$}

As showed in Table XII, an extensive use of dabigatran etexilate would result in:

- A saving for the NHS at the fifth year of observation of 156 million Euros vs. the treatment with VKA $(+27 \%$ compared to the basal hypothesis).

- A reduction of 26,297 in the total number of cumulative strokes vs. the treatment with VKA, with all the obvious social and health benefits in terms of health "saved" (savings in terms of clinical benefit remain unchanged).

\section{Cost of dabigatran etexilate $1.89 € /$ day $(-10 \%$ vs. basal hypothesis)}

As showed in Table XIII an extensive use of dabigatran etexilate would result in:

- In this situation a saving for the NHS at the fifth year of observation of 149 million Euros vs. the treatment with VKA $(+24,6 \%$ compared to the basal hypothesis).

- A reduction of 26,297 in the total number of cumulative strokes vs. the treatment with VKA, with all the obvious social and health benefits in terms of health "saved" (savings in terms of clinical benefit remain unchanged).

\section{Cost of dabigatran etexilate $2.31 € /$ day $(+10 \%$ vs. basal hypothesis)}

As showed in Table XIV an extensive use of dabigatran etexilate would result in :

- Also in this situation a saving for the NHS at the fifth year of observation of 96 million Euros vs. the treatment with VKA ( $-21 \%$ compared to the basal hypothesis).

- A reduction of 26,297 in the total number of cumulative strokes vs. the treatment with VKA, with all the obvious social and health benefits in terms of health "saved" (savings in terms of clinical benefit remain unchanged).

\section{DISCUSSION}

The current treatment of NVAF involves the reduction of only 2,851 strokes, in the first year of observation, compared with the total number of strokes developed in the scenario of total non-treatment $(15,075)$. This confirms that, despite the enormous organizational effort, there is still a major unmet need in the management of NVAF. The underuse of oral anticoagulation, associated with the difficulty in keeping the patients treated with VKA within an acceptable therapeutic range, results in an enormous social and human cost, represented by a total of more than 63,000 strokes cumulated in the 5-year period considered (Table II). The cumulative cost for the current treatment of NVAF over the 5 -year period exceeds $€ 2.3$ billions and the major cost generator in NVAF management is stroke management cost (2.03 billions). Indirect costs, burdening families and society, were not considered in this analysis. According to the CENSIS survey, presented in 2011 at the World Day of stroke, every stroke patient costs about $€ 30,000$ to his/her family per year [18]. The number of avoided strokes per year, resulting from the scenario where all NVAF patients are treated with VKA, amounts to 5,219 compared to no treatment $(-2,368$ compared to the current situation), although the number of events remains high (about 10,000 events/year; Table V). Again, as in the previous scenario, the cumulative cost over the 5 -year period is over 2 billion Euros (2.294 billion Euros). Similarly to the previous scenarios, starting from the cost assumptions reported in the background, and using a daily cost of dabigatran etexilate equal to 2.10 Euros (the lowest in Europe in May 2012: current price in Spain), an extensive use of dabigatran etexilate would cause:

- A reduction of more than 38,500 of the total cumulative number of strokes vs. the current scenario and over 26,297 vs. the treatment with VKA, with all the obvious social and health benefits in terms of health "saved".

- An increase in the cost of drug treatment, which in this case must be considered as the potential maximal expenditure.

- Savings for the NHS at the fifth year of observation of 173 million Euros vs. the current treatment and 123 million Euros vs. treatment with VKA.

The savings from the reduction of indirect costs of both society and families of patients with NVAF should be added to all this, resulting in huge direct and indirect savings for the society. 


\section{CONCLUSIONS}

According to the model used in the present study, which includes both epidemiologic and economic data, it is possible to affirm that marketing dabigatran etexilate in Italy with the new SPAF (stroke prevention in atrial fibrillation) indication is economically sustainable: it allows savings for the NHS in the management of patients with NVAF from the second year vs. no treatment and vs. treatment with VKA. Furthermore it appears socially and ethically convenient as it allows a significant overall increase in expectancy and quality of life of patients with NVAF, through the reduction of the total number of strokes.
Finally dabigatran etexilate allows significant savings in indirect costs for society and families involved in a debilitating disease such as stroke. These costs are inherently difficult to quantify, yet priceless when considering the socio-emotional impact caused by a stroke, not only for the patient but also for all the relatives involved in this important event.

\section{AKNOWLEDGEMENTS}

The authors met criteria for authorship as recommended by the International Committee of Farmaeconomia Health economics and therapeutic pathways and were involved at all stages of manuscript development

\section{REFERENCES}

1. SER - Servizio Epidemiologico Regione Veneto. Available on http://www.ser-veneto.it/index.php

2. Lloyd-Jones DM, Wang TJ, Leip EP, et al. Lifetime risk for development of atrial fibrillation: the Framingham Heart Study. Circulation 2004; 110: 1042-6; doi: 10.1161/01.CIR.0000140263.20897.42

3. ACC/AHA/ESC 2006 Guidelines for the management of patients with atrial fibrillation. Report of the American College of Cardiology/American Heart Association Task Force on Practice Guidelines and the European Society of Cardiology Committee for Practice Guidelines developed in collaboration with the European Heart Rhythm Association and the Heart Rhythm Society. Europace 2006; 8: 651-745; doi: 10.1093/europace/eul097

4. A.L.I.Ce Italia Onlus. Ictus prima causa di disabilità in Italia, 2011. Available on http://www.aliceitalia.org/pagina_news_nazionale.php?id_contenuto_pagina $=69$

5. IMS Health Data, 2011

6. Connolly SJ, Pogue J, Eikelboom J, et al. Benefit of oral anticoagulant over antiplatelet therapy in atrial fibrillation depends on the quality of international normalized ratio control achieved by centers and countries as measured by time in therapeutic range. Circulation 2008; 118: 2029-37; doi: 10.1161/CIRCULATIONAHA.107.750000

7. Connolly SJ, Ezekowitz MD, Yusuf S, et al.; RE-LY Steering Committee and Investigators. Dabigatran versus warfarin in patients with atrial fibrillation. $N$ Engl J Med 2009; 361: 1139-51; doi: 10.1056/NEJMoa0905561

8. Friberg L, Rosenqvist M, Lip GY. Evaluation of risk stratification schemes for ischaemic stroke and bleeding in 182678 patients with atrial fibrillation: the Swedish Atrial Fibrillation cohort study. Eur Heart J 2012 [Epub ahead of print]; doi: 10.1093/eurheartj/ehr488

9. Hart RG, Pearce LA, Aguilar MI. Meta-analysis: antithrombotic therapy to prevent stroke in patients who have nonvalvular atrial fibrillation. Ann Intern Med 1999; 131: 492-501

10. Connolly SJ, Ezekowitz MD, Yusuf S, et al., Randomized Evaluation of Long-Term Anticoagulation Therapy Investigators. Newly identified events in the RE-LY trial. N Engl J Med 2010; 363: 1875-6; doi: 10.1056/NEJMc1007378

11. Degli Esposti L, Sangiorgi D, Di Pasquale G, et al. Adherence to treatment and anticoagulation control in vitamin $\mathrm{K}$ antagonists-treated patients: an administrative databases analysis in a large Italian population. Farmaeconomia e Percorsi Terapeutici 2011; 12: 69-75

12. White HD, Gruber M, Feyzi J, et al. Comparison of outcomes among patients randomized to warfarin therapy according to anticoagulant control: results from SPORTIF III and V. Arch Intern Med 2007; 167: 239-45; doi: 10.1001/archinte.167.3.239

13. Gerzeli S, Tarricone R, Zolo P, et al. The economic burden of stroke in Italy. The EcLIPSE Study: Economic Longitudinal Incidence-based Project for Stroke Evaluation. Neurol Sci 2005; 26: 72-80; doi: 10.1007/s10072-005-0439-0

14. Legge regionale Regione Veneto 11/2008, n.14, Bur n. 93 del 11/11/2008. Misure per migliorare la qualità della vita dei pazienti in terapia anticoagulante

15. Go AS, Hylek EM, Phillips KA, et al. Prevalence of diagnosed atrial fibrillation in adults: national implications for 
rhythm management and stroke prevention: the AnTicoagulation and Risk Factors in Atrial Fibrillation (ATRIA) Study. JAMA 2001; 285: 2370-5; doi: 10.1001/jama.285.18.2370

16. Camm AJ, Kirchhof P, Lip GY, et al.; European Heart Rhythm Association; European Association for CardioThoracic Surgery. Guidelines for the management of atrial fibrillation: the Task Force for the Management of Atrial Fibrillation of the European Society of Cardiology (ESC). Eur Heart J 2010; 31: 2369-429

17. Roskell NS, Lip GY, Noack H, et al. Treatments for stroke prevention in atrial fibrillation: A network meta-analysis and indirect comparisons versus dabigatran etexilate. Thromb Haemost 2010; 104: 1106-15; doi: 10.1160/TH10$10-0642$

18. The social costs and the care needs of patients with stroke. CENSIS Survey, 2010

\section{APPENDIX A}

Figure 1A shows the basis for the calculation of patients with non-valvular atrial fibrillation (NVAF) eligible for treatment

\section{Reference}

1. Osservatorio Epidemiologico Cardiovascolare Italiano

2. SER - Servizio Epidemiologico Regione Veneto. Available on http://www.serveneto.it/index.php

3. ISTAT - Resident Population in Italy by age, sex, and marital status on 1 January 2011. Available on http://demo.istat.it/

4. ATA-AF Study - AntiThrombotic Agents in Atrial Fibrillation. $42^{\circ}$ Congresso Nazionale di Cardiologia dell'Associazione Nazionale Medici Cardiologi Ospedalieri (ANMCO)

5. IMS Health Data, 2011

6. Survey SIMG-ANMCO. National Congress SIMG, 2011

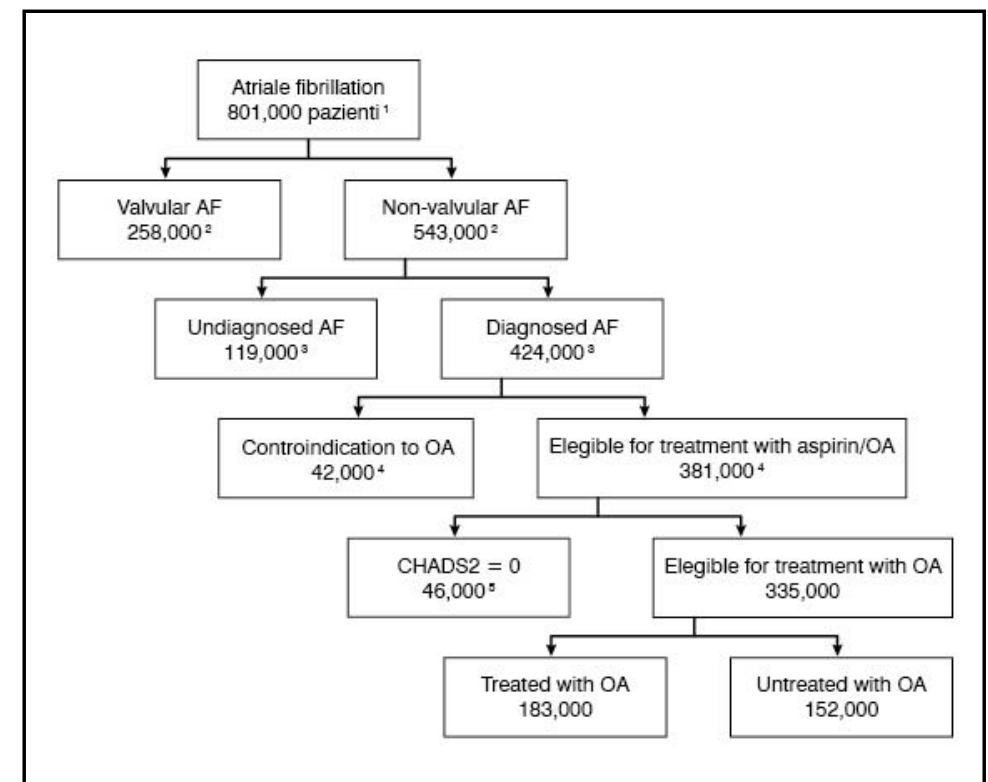

Figure 1A. Bases for the calculation of patients with (NVAF) eligible for treatment ${ }^{1} \mathrm{AF}$ based on data from Osservatorio Epidemiologico Cardiovascolare [1] and Servizio Epidemiologico della Regione Veneto [2] applied to the Italian population, ISTAT 2011 [3]

${ }^{2} 32.2 \%$ cases of valvular AF; from ATA-AF study by ANMCO and FADOI 2011 [4]

${ }^{3} 22 \%$ are undiagnosed; from market research IMS 2010 [5]

${ }^{4} 10 \%$ with contraindication to OA; conservative estimate compared to $22 \%$ recorded in ATA-AF study by ANMCO and FADOI 2011 [4]

${ }^{5} 12 \%$ CHADS = 0; from survey SIMG 2011 [6] 